\title{
Experimental study of a helicopter rotor model in hover
}

\author{
Filip Panayotov ${ }^{1}$, Ivan Dobrev², Fawaz Massouh ${ }^{2}$, and Michael Todorov ${ }^{1, *}$ \\ ${ }^{1}$ Technical University of Sofia, Department of Air Transport, 8 Kliment Ohridski Blvd., 1000 Sofia, Bulgaria \\ ${ }^{2}$ Arts et Métiers - ParisTech, DynFluid laboratory, blvd. L’Hôpital 151, 75013 Paris, France
}

\begin{abstract}
This paper presents the results from an experimental study of the aerodynamic performance and efficiency of a model helicopter rotor in steady hover at Reynolds numbers below 70000. Results are shown for a two-, three- and four-bladed rotor configuration for various pitch angles and speeds of rotation. The influence of the rotor solidity on the aerodynamic efficiency in terms of the Figure of Merit is highlighted. The profile drag component is evaluated as a function of the Reynolds number. The internal friction losses of the test stand are estimated and taken into account in all measurements. A brief description of the designed test stand is provided. The experimental setup allows for the measurement of thrust and torque of helicopter rotors with diameters of up to 1 meter, for pitch angles varying from $-12^{\circ}$ to $+24^{\circ}$ and for rotational speeds of up to 3000 RPM. Conclusions are drawn about the aerodynamic performance and efficiency of the studied rotor configurations. This experimental study provided a significant database, which will serve for validation purposes.
\end{abstract}

\section{Introduction}

In recent years, there has been a significant increase in the number of studies on the aerodynamic performance of small-scale helicopter rotors of unmanned aerial vehicles (UAVs). This is largely due to the recent increase of usage of UAVs for various commercial, leisure and military applications.

Although there is a large amount of data available from decades-long theoretical, numerical and experimental studies of full scale helicopter rotors, it is not applicable in cases of validation of newly developed models for low-Reynolds flows, due to the existing particularities regarding the aerodynamic performance of scaled rotors. Their overall efficiency, which in the case of hover is expressed with the Figure of Merit (FM), is reduced in low-Reynolds flow conditions.

According to [1-3] the following particularities apply for those flow conditions:

- the boundary layer is thicker and susceptible to flow separation at small angles of attack,

- the profile drag is significantly bigger as a share in percentage from the induced drag when compared with a full-scale rotor with the same geometry,

- the swirl effects on the flow downstream of the rotor are more pronounced than those of the full-scale rotors.

Different approaches are used to study the aerodynamic performance of UAV rotors. They can be classified into the following three main groups, namely:

- Classic aerodynamic theory,

- CFD simulations,

- Experimental studies.

The classic aerodynamic solutions are based on existing theories, initially developed for airscrews, which were later adapted for helicopter rotors. Those include: the Actuator Disk Theory (ADT); the Blade Element Theory (BET); the Blade Element Momentum Theory (BEMT); the prescribed-vortex wake method and the free-vortex wake method, adapted for helicopter rotors in hover [4].

Paper [5] is a good example of the capabilities of the free-vortex wake methods not only to precisely simulate the hover performance of helicopter rotors but also to allow for the study of unsteady flow phenomena.

Computational fluid dynamics (CFD) is based on the numerical resolution of inviscid Euler equations or Navier-Stokes equations with an appropriate turbulence model, in order to determine the parameters of the flowfield in the simulated region around the studied rotor. This approach provides a more precise estimation of the aerodynamic (pressure) loads distribution along the blades. Although computationally expensive, the CFD simulations allow for a more detailed study of the helicopter rotor aerodynamics. Those simulations are very useful when studying unsteady rotor operation. In such a way the interaction between the tip-vortices and the rotor blades can be examined, which in return provides a more precise estimation of the aerodynamic performance of the rotor.

The hybrid CFD models offer a good compromise between high degree of accuracy of the obtained solution and reduced amount of computational resources. The most common approach is to replace the studied rotor geometry in the CFD model with the equivalent surface or volume distributed forces. This results in a significant reduction in the complexity of the simulation. The authors in [6] have applied this approach in their study, which allowed them to evaluate the rotor performance, while

\footnotetext{
*Michael Todorov: Michael.Todorov@tu-sofia.bg
} 
taking into account the complex dynamics of the lead-lag, torsion and flapping motions of the blades.

Each of the above mentioned approaches has its advantages and disadvantages in terms of the accuracy of the obtained results, the required computational resources and the necessary simulation time. In principle, all numerical models need to be validated against experimental data. Then only a validated solution can be used for aerodynamic performance studies or geometry optimization of UAV rotors.

Thus, experimental studies are performed in order to obtain large databases for validation and calibration purposes of newly developed numerical models. Due to the absence of experimental data for small-scale propellers, operating at low-Reynolds flow conditions, it is not a privilege but rather a necessity for universities and companies developing UAVs to possess their own test stands for rotors and propellers [7-9]. These facilities allow not only for the acquisition of experimental data but also provide the possibility to test commercially available model rotors and propellers, as well as to assess new designs and concepts. They provide the opportunity for research and development activities.

In [10] the authors evaluate the hover performance of a helicopter rotor. For this purpose a rotor test stand is built. The authors found out that while the scaled rotors operate in a low-Reynolds environment, there is between 15 to $20 \%$ lower aerodynamic efficiency in hover as compared to a full-scale rotor with the same rectangular untwisted blade with NACA 0015 aerodynamic profile.

The aim of the present work is to evaluate experimentally the aerodynamic performance of scaled helicopter rotors. This study will also reveal the capabilities of the specialized test stand, developed in the Department of Aeronautics of Technical University Sofia. The other main objective of this work is to acquire a large experimental database, which will serve for validation and calibration of numerical solutions under development.

\section{Experimental studies}

With the use of a specific helicopter rotor test facility, different series of measurement for multiple speeds of rotation and pitch angles are performed in order to study the aerodynamic characteristics and hover efficiency of three rotor configurations with 2, 3 and 4 blades.

\subsection{Experimental setup}

Fig. 1 shows the helicopter rotor test stand, which provides the possibility to measure the produced thrust $T, \mathrm{~N}$, the torque $Q, \mathrm{Nm}$ and the angular velocity $\Omega, \mathrm{rad} / \mathrm{s}$ of scaled helicopter rotors with diameters of up to 1 meter and pitch angles varying from $-12^{\circ}$ to $+24^{\circ}$. The following measuring devices are used:

- Torque transducer HBM T20WN with nominal (rated) torque of $1 \mathrm{Nm}$, accuracy class 0.2 and output voltage $\pm 10 \mathrm{~V}$,

- Force transducer HBM S2M with nominal (rated) force of $10 \mathrm{~N}$, accuracy class: 0.02 and output voltage of $2 \mathrm{mV} / \mathrm{V}$.

The scaled rotors are driven with a brushless electric motor Maxon ECi-40 with nominal (rated) power of $100 \mathrm{~W}$. It allows for rotational speeds of up to $4190 \mathrm{RPM}$, although the maximum permissible rotational speed is limited to 3000 RPM. The electric motor has built-in Hall sensors, which make it possible to measure the speed of rotation. The motor is driven by Maxon ESCON 50/5 servo controller, which allows for precise regulation of the rotational speed.

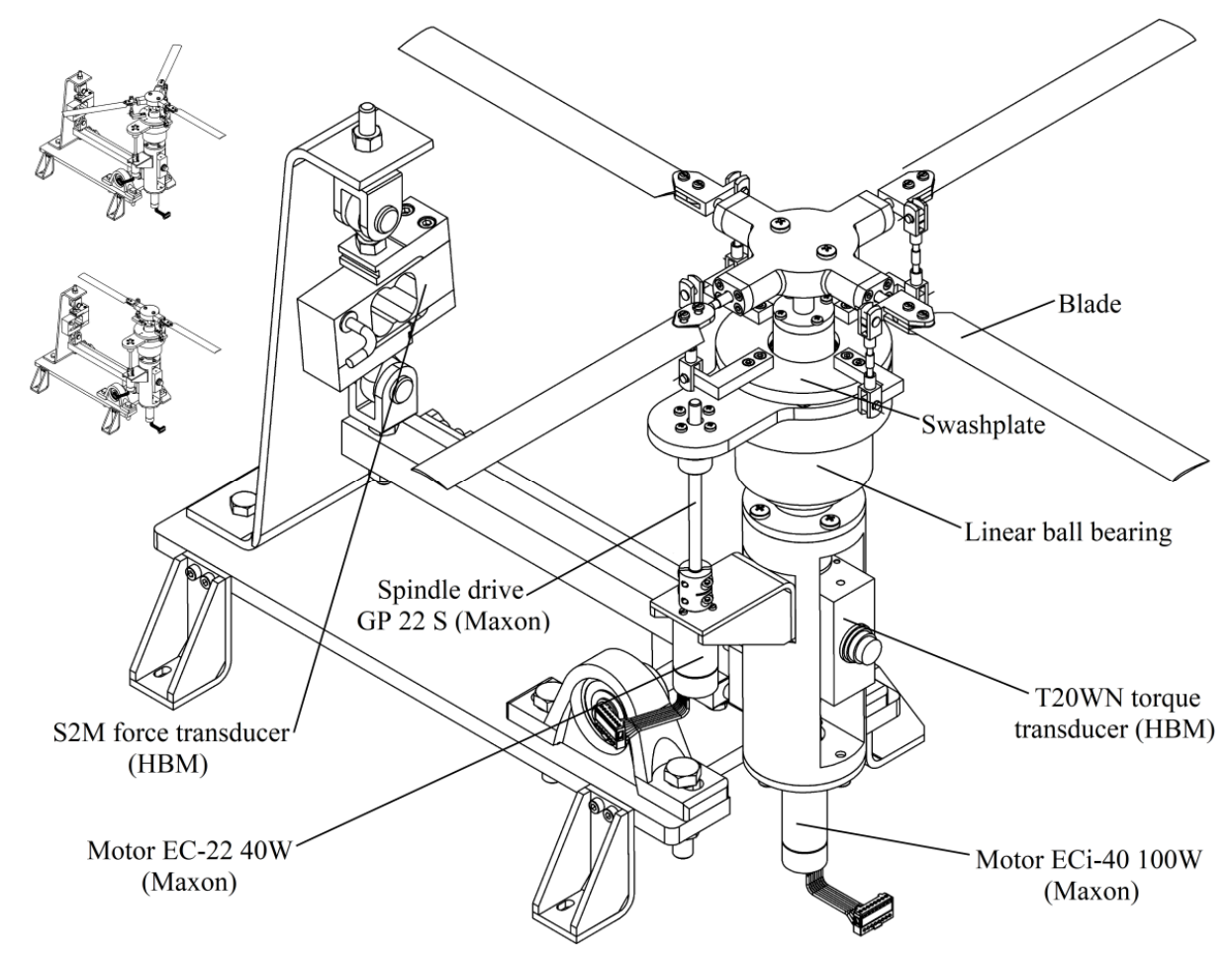

Fig. 1. Helicopter rotor test stand. 
The test stand allows for the variation of the pitch angle of the blades. This is achieved by a spindle-driven mechanism, which uses the Maxon GP22S spindle with gear ratio of 1:370 and the EC-22 electric motor with nominal (rated) power of $40 \mathrm{~W}$. This motor is also controlled by the ESCON 50/5 servo controller and can achieve a rate of change of $\pm 3 \%$.

The pitch angle may be varied during rotation of the helicopter rotor. Thus, unsteady processes can be studied, in order to evaluate their impact on the aerodynamic characteristics of the rotor.

A specially developed LabVIEW driver permits both electric motors to be controlled automatically according to a pre-programmed test schedule. Thus, multiple operational regimes can be evaluated in one test session without the need to constantly stop, re-program and restart the test bench. An automatic data acquisition system is organized, which stores the experimental data in a wellstructured database.

The data acquisition from the force and torque transducers is ensured by the HBM PMX measuring amplifier system, shown in Fig.2. The PMX module is equipped with CODESYS V3 Soft-PLC, which allows for easy setup, configuration and calibration of each individual channel. It offers various analogue and digital inputs and outputs, thus providing large data acquisition capacity and control flexibility.

The entire test bench is powered by a DC power supply HY3005D2, which provides up to $300 \mathrm{~W}$.

The construction of the test stand allows for easy and rapid change of the rotor configuration. The test stand can easily be modified for the attachment of hinged rotors.

\subsection{Studied rotors}

The present study concerns three-rotor configurations, differing in the number of blades, such as shown in Fig. 3. All three rotor configurations are hingeless with an inner diameter of $0.162 \mathrm{~m}$ and an outer diameter of $0.532 \mathrm{~m}$. The blades are rectangular and untwisted with a chord of 0.025 $\mathrm{m}$. The blade profile is Goettingen 417 (GOE417).

\subsection{Methodology}

Three experimental studies were organized and carried out. The first one aimed to evaluate the torque $Q_{n b}$ due to internal friction losses in the bearings of the main shaft and the air friction, created by the undetectable rotor hub. These measurements are important as they are later subtracted from all acquired torque data in order to deduce the real rotor torque without overestimation. The second one aims to estimate the coefficient of profile drag at zero thrust $C_{D 0}$. This study is performed only with the twobladed rotor configuration as it permits higher rotational speed and thus a broader range of Reynolds numbers can be reached. In this case the torque $Q_{0}$ is registered only if $T=0 \mathrm{~N}$.

In order to obtain the $C_{D 0}$, equations (1), (2) and (3) are used [4]:

$$
\begin{gathered}
C_{p d}=\frac{\left(Q_{0}-Q_{n b}\right) \Omega}{\rho A \Omega^{3} R^{3}}=\frac{Q_{p d} \Omega}{\rho A \Omega^{3} R^{3}}=\frac{P_{p d}}{\rho A \mathrm{~V}_{t i p}^{3}}, \\
C_{D 0}=\frac{8 C_{p d}}{\sigma}, \\
\sigma=\frac{N_{b} c}{\pi R},
\end{gathered}
$$

where $\rho$ is the air density of $1.225 \mathrm{~kg} / \mathrm{m}^{3} ; A=\pi R^{2}, \mathrm{~m}^{2}$ is the area of the rotor; $\Omega, \mathrm{rad} / \mathrm{s}$ is the angular speed of rotational; $R, \mathrm{~m}$ is the radius of the rotor; $V_{\text {tip }}=\Omega R, \mathrm{~m} / \mathrm{s}$ is the tip speed of the blades; $N_{b}$, - is the number of blades of the rotor configuration; $c, \mathrm{~m}$ is the blade chord; $\sigma$, - is the rotor solidity; $Q_{p d}=Q_{0}-Q_{n b}, \mathrm{Nm}$ is the rotor torque due to profile drag; $P_{p d}=Q_{p d} \Omega$, W is the power consumption due to profile drag; and $C_{p d}$, - is the coefficient of profile drag force.

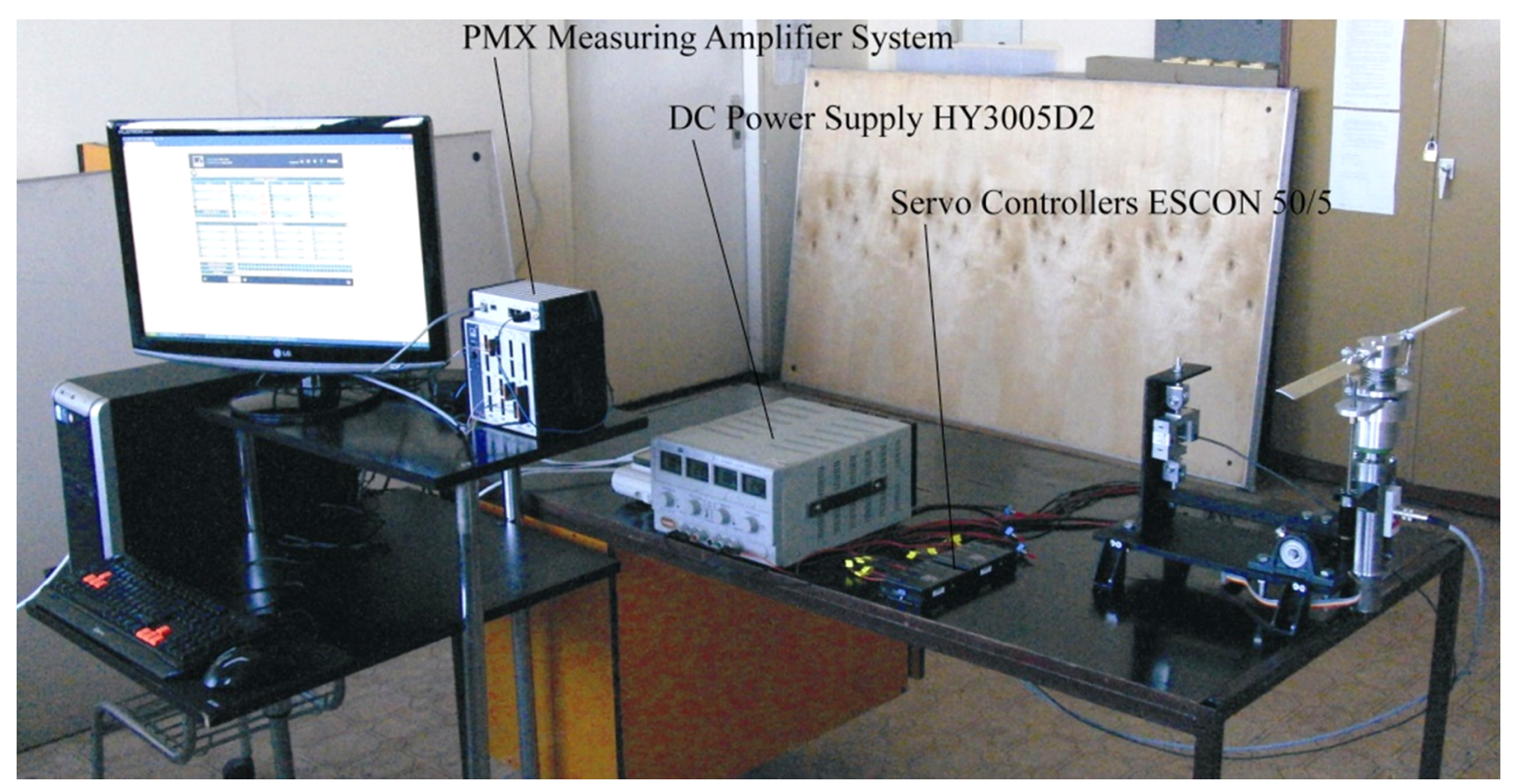

Fig. 2. Helicopter rotor test stand with the two-bladed rotor configuration. 


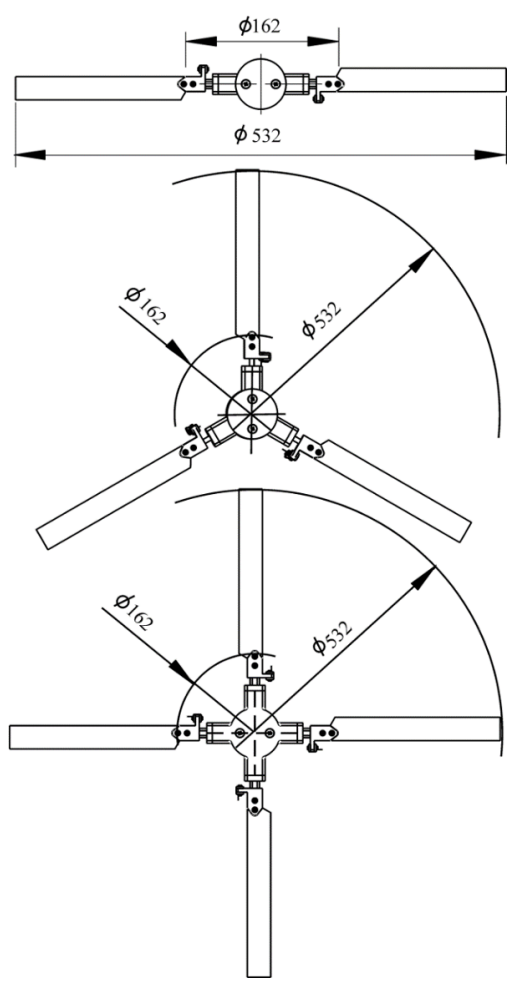

Fig. 3. Studied rotor configuration (dimensions in millimetres). Blade airfoil: GOE417; Blade chord: $25 \mathrm{~mm}$.

The third study is such that for each rotor configuration a fixed pitch angle is set and then the thrust $T, \mathrm{~N}$ and torque $Q, \mathrm{Nm}$ are measured for a multiple set of rotational speeds. This procedure is repeated for all preselected pitch angles $\theta=0^{\circ}, 3^{\circ}, 6^{\circ}, 9^{\circ}, 12^{\circ}$ and $15^{\circ}$.

In [4] the coefficient of thrust $C_{T}$ is given with (4); the coefficient of power $C_{P}$ is given with (5); and the Figure of Merit $(F M)$ is defined with (6):

$$
\begin{gathered}
C_{T}=\frac{T}{\rho A \Omega^{2} R^{2}}, \\
C_{P}=\frac{Q \Omega}{\rho A \Omega^{3} R^{3}}=\frac{P}{\rho A \Omega^{3} R^{3}}, \\
F M=\frac{\mathrm{C}_{T}^{3 / 2}}{\sqrt{2} C_{P}} .
\end{gathered}
$$

In [4] the Figure of Merit computed with (6) is shown to have been introduced as a nondimensional measure of hovering thrust efficiency for helicopter rotors, which provides a common base for comparison between rotors with different geometries.

In order to ensure comparability between the results for helicopter rotors with different number of blades and thus with different rotor solidity, the following dimensionless parameters are used:

- $C_{T} / \sigma$, - - blade loading coefficient,

- $C_{P} / \sigma,--$ power coefficient.

\subsection{Experimental results}

Fig.4. shows the decomposition of the rotor torque for the two-bladed configuration at zero thrust. From Fig. 4 it can be seen that initially the profile drag torque is negligible right up to 400 RPM, after which it grows significantly. It can be seen that between 800 and 1300 RPM the profile torque $Q_{p d}$ equalizes its value with the no blades torque $Q_{0}$, which is due to internal frictions in the main shaft bearings. After 1400 RPM the profile drag torque rises as a curve of second order, where the no blade torque is rather linear.

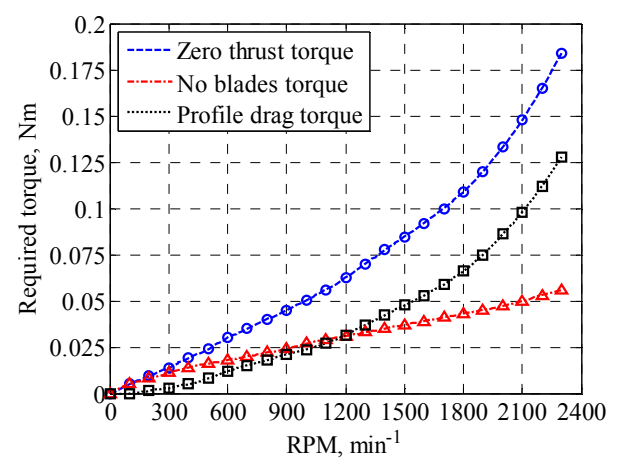

Fig. 4. Evaluation of the torque required to drive the test stand at zero thrust for the two-bladed rotor at various speeds.

The data in Fig.5 is derived from Fig.4. by using the simple relation between torque and power: $P=Q \Omega$. It can be seen that at 1000 RPM the power losses, due to bearing frictions, are around $3 \mathrm{~W}$, where at 2000 RPM they rise to $10 \mathrm{~W}$. At $1000 \mathrm{RPM}$ the total power consumption at zero thrust is about $5 \mathrm{~W}$.

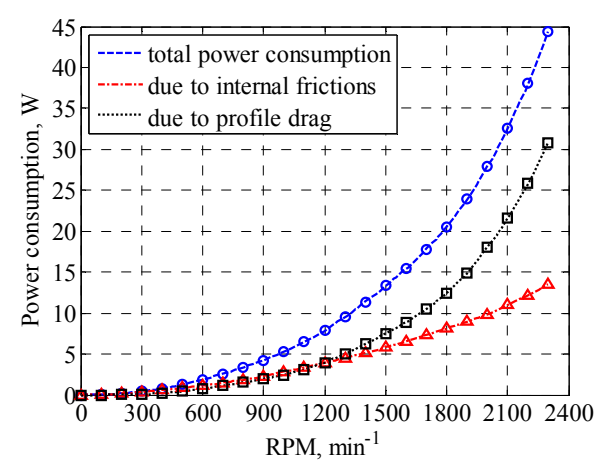

Fig. 5. Estimated power consumption required to drive the test stand at zero thrust for the two-bladed rotor at various speeds.

Fig. 6. shows the variation of the profile drag at zero thrust $C_{D 0}$ against the Reynolds number of the flow. Those results are closely comparable to those published in [11]. It can be seen that the profile drag reduces with $35 \%$ with the increase of Re from 20000 to 50000.

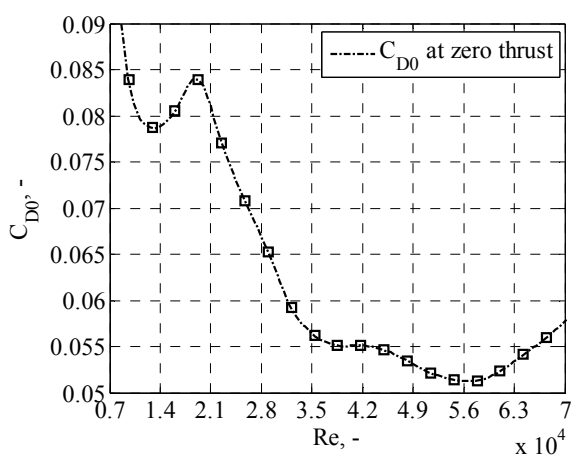

Fig. 6. Profile drag at zero thrust depending on Reynolds for profile Goettingen 417. 
Fig.7. shows the hover polar for all rotor configurations. It can be seen that the three and four bladed-rotor configurations require lower hover power.

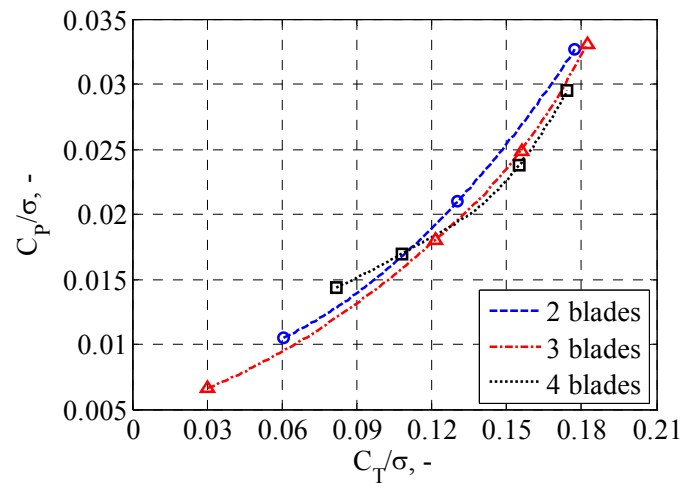

Fig. 7. Hover polar for all rotor configurations at 900 RPM and $\mathrm{Re} \approx 29000$.

Fig.8. shows the blade loading coefficient variation from the pitch angle of the rotor blades $\theta$. It can be seen that the lower the number of blades, the higher the blade load on each individual blade, which may lead to early stall conditions as it can be observed for the two-bladed configuration for $\theta=12^{\circ} \div 15^{\circ}$.

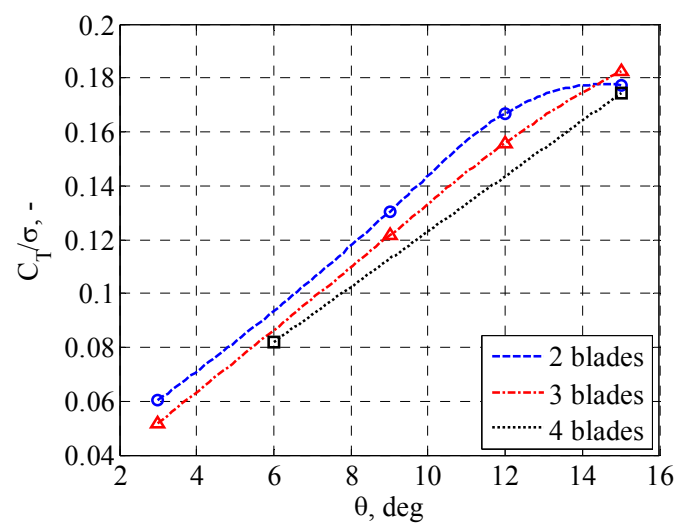

Fig. 8. Blade loading coefficient against the pitch angle of the blades for all rotor configurations at 900 RPM and Re $\approx 29000$.

Fig.9. shows the hover efficiency against the blade load coefficient. It is clear that the higher the number of blades, the higher the rotor solidity and thus the higher the hover efficiency of the rotor.

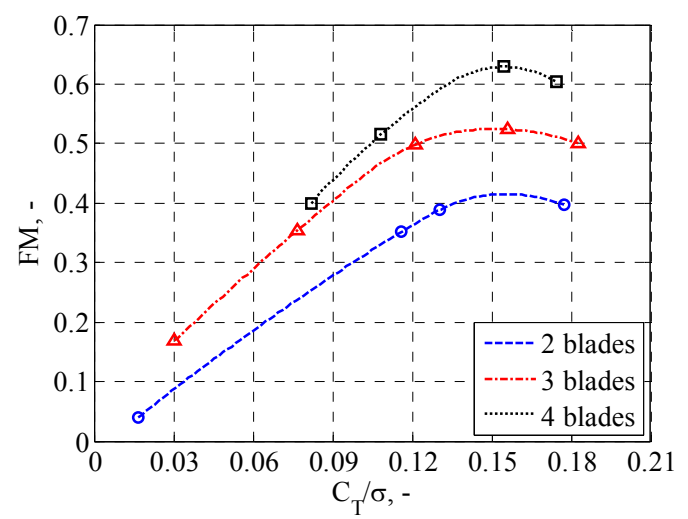

Fig. 9. Figure of Merit against the blade loading coefficient for all rotor configurations at $900 \mathrm{RPM}$ and $\mathrm{Re} \approx 29000$.

\section{Summary and conclusions}

This paper presents an experimental approach for the study of a helicopter rotor model at low-Reynolds flow conditions.

A fully operational test stand is designed and shown. It allows for the evaluation of the aerodynamic performance and efficiency of model helicopter rotors in steady hover. Thus, three rotor configurations with 2, 3 and 4 blades were considered.

Thanks to this study, a relatively large database was obtained. This experimental data is primarily for lowReynolds flow conditions of hovering rotors. It will be quite useful for validation and calibration purposes in the case of newly developed numerical solutions.

The obtained results required the implementation of an important experimental and technical know-how.

\section{References}

1. M. Benedict, J. Winslow, Z. Hasnain, I. Chopra, Performance and flowfield measurements of a MAVscale helicopter rotor in hover, Int. J. of Micro Air Vehicle, 7(3), 231-255 (2015)

2. B. Hein, I. Chopra, Hover performance of a micro air vehicle: Rotors at low Reynolds number, J. of the American Helicopter Society, 52(3), 254-262 (2007)

3. M. Ramasamy, B. Johnson, J. Leishman, Understanding the aerodynamic efficiency of a hovering micro rotor, J. of the American Helicopter Society, 53(4), 412-428 (2008)

4. J. Leishman, Principles of Helicopter Aerodynamics, 67-70 (Cambridge university press, 2006)

5. A. Karpatne, J. Sirohi, S. Mula, C. Tinney, Vortex ring model of tip vortex aperiodicity in a hovering helicopter rotor, J. of Fluids Engineering, 136 (2014)

6. I. Dobrev, F. Massouh, M. Todorov, A hybrid aerodynamic model for a study of pattern helicopter rotor dynamics, NCAS-2013, 11-14 (2013)

7. J. Brandt, M. Selig, Propeller performance data at low Reynolds numbers, AIAA 2011-1255 (2011)

8. V. Serbezov, S. Dimitrov, K. Rangelov, Mobile test stand for evaluation of electric power plants for unmanned aircraft, BulTrans-2017, MATEC Web of Conferences, 133 (2017)

9. H. Panayotov, S. Pentchev, Determination of propeller characteristics for multirotor drone design, TECHSYS2017, 264-268 (2017)

10. B. Lee, Y. Byun, J. Kim, B. Kang, Experimental hover performance evaluation on a small-scale rotor using a rotor test stand, J. of Mech. Science and Technology, 25(6), 1449-1456 (2011)

11. C. Lyon, A. Broeren, P. Giguere, A. Gopalarathnam, M. Selig, Summary of low-speed airfoil data, 3, 128132 (1995) 